\title{
Low bending loss waveguide opens the avenue to downsizing of 3D photonic integrated circuits
}

\author{
YongFeng $\mathrm{Lu}^{*}$ \\ Department of Electrical and Computer Engineering, University of Nebraska, Lincoln NE 68588-0511, USA
}

Received March 12, 2018; accepted March 14, 2018; published online March 21, 2018

Citation: Y. F. Lu Low bending loss waveguide opens the avenue to downsizing of 3D photonic integrated circuits, Sci. China-Phys. Mech. Astron. 61, 070331 (2018), https://doi.org/10.1007/s11433-018-9203-2

Photonic integrated circuits (PIC) enable efficient and flexible control of photons within a compact space, thereby revolutionizing both information technology and biological and chemical sensing. Currently, semiconductor materials including silicon, silicon nitride ( $\mathrm{SiN}$ ), and indium phosphate (InP) are still the prominent PIC platforms, which can be patterned using mature photolithographic technologies [1]. Being an intrinsically planar fabrication technology, increasing the integration density in the photolithography mainly depends on reducing the sizes of individual components.

Alternatively, PICs of geometrically complex three-dimensional (3D) configurations can now be fabricated using femtosecond-laser direct writing, which potentially provides higher integration density and more flexibility in terms of integrated multifunctional systems such as optofluidics and optomechanics $[2,3]$. The principle of femtosecond laser fabrication of PICs is illustrated as follows.

As shown in Figure 1, permanent changes in the refractive index can be induced only in the focal volumes of tightly focused femtosecond laser pulses due to the high-order nonlinear interaction between the femtosecond laser beam and the substrate. Thus, scanning the focal spot within the substrate along a predefined path will produce a modification track with an increased refractive index, which acts as an optical waveguide [4]. In particular, the combination of

*Corresponding author (email: ylu2@unl.edu) femtosecond laser waveguide writing and high purity fused silica of very low absorption loss has been proven effective for producing high quality single-mode waveguides of propagation losses as low as $\sim 0.2 \mathrm{~dB} / \mathrm{cm}$ [5], which has enabled a variety of applications ranging from quantum information processing to optical communication systems. Nevertheless, for the technique to become a mature industrial platform, one problem is yet to be solved. The typical refractive index increase induced in fused silica by femtosecond laser irradiation is on the order of $\sim 10^{-4}-10^{-3}$, giving rise to large bending losses at small radii of curvatures. This has become a major obstacle for producing compact photonic devices with the 3D waveguides written by femtosecond laser pulses.

Recently, Liu et al. [6] reported a technique which reduces the bending loss at small radii of curvatures by more than one order of magnitude. The key idea of their approach is shown in Figure 2. An optical waveguide of low propagation loss is

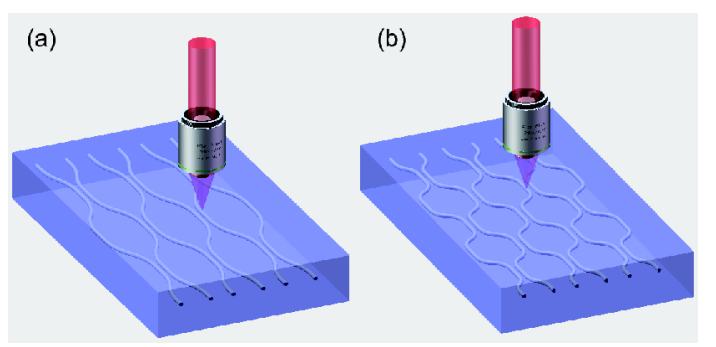

Figure 1 (Color online) Concept of writing 3D PICs using a femtosecond laser. The PIC in (a) shows a lower integration density than that in (b) due to the larger bending radius of the waveguides in (a). 


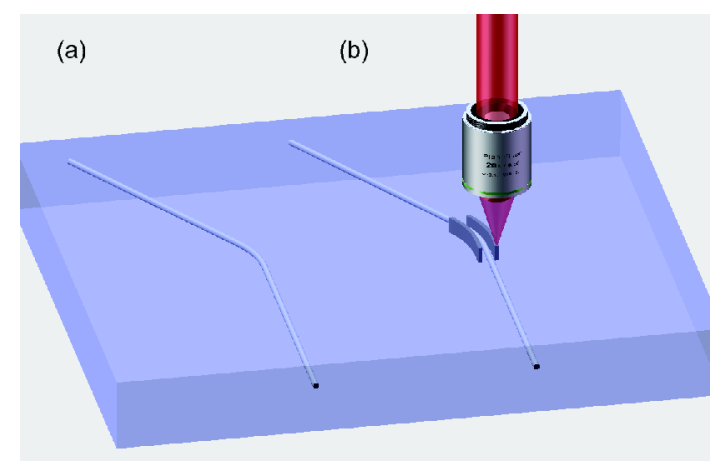

Figure 2 (Color online) Femtosecond laser writing of curved waveguides (a) without and (b) with BLSWs.

first inscribed in fused silica by optimizing the laser conditions, as shown in Figure 2(a). However, such optimal conditions for minimizing the loss in the straight waveguide cannot provide sufficient refractive index contrast for completely confining the light in curved segments of the waveguide. To circumvent this problem, Liu et al. additionally write two sidewalls, i.e., the so-called bending-loss-suppression walls (BLSWs), along the bend of the waveguide, as shown in Figure 2(b). The stress generated by the BLSWs dramatically promotes the refractive index increase within the waveguide, i.e., the refractive index contrast between the waveguide and its surrounding area is increased almost by a factor of 2, namely, from $1.85 \times 10^{-3}$ to $3.45 \times 10^{-3}$. As a result, the bending loss of the waveguide with a radius of curvature of $15 \mathrm{~mm}$ decreases from 3-4 to $\sim 0.3 \mathrm{~dB}$. Since small radii of curvatures benefit large-scale integration of PICs on a single chip, it is expected that the technique can be highly valuable for construction of chip-based photonic devices.

1 B. Jalali, and S. Fathpour, J. Lightw. Technol. 24, 4600 (2006).

2 K. Sugioka, and Y. Cheng, Lab Chip 12, 3576 (2012).

3 B. Lenssen, and Y. Bellouard, Appl. Phys. Lett. 101, 103503 (2012).

4 K. M. Davis, K. Miura, N. Sugimoto, and K. Hirao, Opt. Lett. 21, 1729 (1996).

5 Y. Nasu, M. Kohtoku, and Y. Hibino, Opt. Lett. 30, 723 (2005).

6 Z. M. Liu, Y. Liao, Z. W. Fang, W. Chu, and Y. Cheng, Sci. ChinaPhys. Mech. Astron. 61, 070322 (2018). 